\title{
A NOTE ON THE PUNCTURED NEIGHBOURHOOD THEOREM
}

\author{
by ROBIN HARTE and WOO YOUNG LEE $\dagger$
}

(Received 19 December, 1995, revised 10 September, 1996)

The punctured neighbourhood theorem can be interpreted as saying that if $0 \in \mathbf{C}$ is on the boundary of the spectrum of a Fredholm operator then it must be an isolated point of that spectrum. This extends to semi-Fredholm operators, in particular to operators with closed range and finite dimensional null space. In this note we generalise both the finite dimensionality of the null space and the scalars involved in the definition of an isolated point of the spectrum.

If $T$ is a linear operator on a vector space $X$ we shall write

$$
T^{\infty}(X)=\bigcap_{n=1}^{\infty} T^{n}(X)
$$

for the hyperrange and

$$
T^{-\infty}(0)=\bigcup_{n=1}^{\infty} T^{-n}(0)
$$

for the hyperkernel of $T$ : it is clear that both subspaces are invariant under any operator $S$ on $X$ which commutes with $T$, although for bounded operators on Banach spaces neither need be closed. If $S$ commutes with $T$ we shall write

$$
S^{\wedge}: T^{\infty}(X) \rightarrow T^{\infty}(X)
$$

for the operator induced by $S$. If also $S$ is invertible then by $[3,7.8 .3 .4]$

$$
(T-S)^{-1}(0) \subseteq T^{\infty}(X),
$$

so that the null space of $T-S$ is the same as that of $(T-S)^{\wedge}$. It is also well known that if the null space of $T$ is finite dimensional then the restriction of $T$ to its hyperrange is onto. which

1. Lemma. If $T$ is bounded and linear on a Banach space $X$ and if there is $k \in \mathbf{N}$ for

1.1

$$
\operatorname{dim} T^{-1}(0) \cap T^{k}(X)<\infty
$$

then

$$
T^{\infty}(X) \subseteq T\left(T^{\infty}(X)\right)
$$

Proof. If $y \in T^{\infty}(X)$, there is $\left(x_{n}\right)$ in $X$ for which $y=T^{n} x_{n}$, for each $n \in \mathbf{N}$. Now if $T^{-1}(y) \cap T^{k}(X)$ is finite dimensional, then there is $N \geq k$ (depending on $y$ ) for which

$$
x_{N} \in T^{-1}(y) \cap T^{N}(X)=T^{-1}(y) \cap T^{\infty}(X) .
$$

† This author was supported in part by research grant BSR1-95-1420.

Glasgow Math. J. 39 (1997) 269-273. 
If the null space of an operator is finite dimensional and the range is closed, then all the powers of $T$ have closed range. Laursen and Mbekhta [7] have noticed that this is also true when the null space is "disjoint" from the range; their argument ([7, Corollary], [4, Theorem 1]) extends to that of finite dimensional intersection.

2. Lемma. If $T: X \rightarrow Y$ and $S: Y \rightarrow Z$ are bounded and linear between Banach spaces, then

$2.1 S(Y)$ and $S^{-1}(0)+T(X)$ closed $\Rightarrow S T(X)$ closed $\Rightarrow S^{-1}(0)+T(X)$ closed

and

2.2 $S^{-1}(0)+T(X)$ closed and $S^{-1}(0) \cap T(X)$ finite dimensional $\Rightarrow T(X)$ closed.

Proof. Most of this is set out in Theorem 1 of [4]. The first part of (2.1) is a lemma of Kato [5, Lemma 331], valid whether or not the space $X$ is complete, and the second part is elementary. When the intersection $S^{-1}(0) \cap T(X)$ is $\{0\}$ then (2.2) is a simple application of the open mapping theorem ([3, Theorem 4.8.2], [7]); if more generally it is finite dimensional consider the operator $W: Z=S^{-1}(0) \times X / T^{-1}(0) \rightarrow Y$ defined by setting

$$
W\left(y, x+T^{-1}(0)\right)=y+T z \text { for each } y \in Y, x \in X .
$$

Evidently $W$ is well-defined, linear, bounded, and onto with finite dimensional null space: hence there is $W^{\prime}: Y \rightarrow Z$, also bounded and linear, for which $W=W W^{\prime} W$. Now observe that

$2.4 E=T W^{\prime}: Y \rightarrow Y$ satisfies $E=E^{2}$ and $T(X)=E(Y)=(I-E)^{-1}(0) \quad$ is closed.

We need also a modification of the second part of (2.1).

3. Lemma. If $S: Y \rightarrow X$ and $T: X \rightarrow Y$ are bounded and linear between Banach spaces, then

$$
T^{-1}(0)+S T(X) \text { closed } \Rightarrow(T S)^{-1}(0)+T(X) \text { closed }
$$

Proof. If $y \in \operatorname{cl}\left((T S)^{-1}(0)+T(X)\right)$, then there are sequences $\left(z_{n}\right)$ and $\left(x_{n}\right)$ such that $z_{n}+T x_{n} \rightarrow y$ with $T S z_{n}=0$, giving

$$
S y=\lim _{n}\left(S z_{n}+S T x_{n}\right) \in \operatorname{cl}\left(T^{-1}(0)+S T(X)\right) .
$$

Since $T^{-1}(0)+S T(X)$ is closed, $S y \in T^{-1}(0)+S T(X)$ and hence $y \in(T S)^{-1}(0)+T(X)$.

The case $T=I$ shows that (3.1) cannot be reversed. The punctured neighbourhood theorem now follows.

4. THEOREM. If $T$ is bounded and linear on a Banach space $X$ with $k \in \mathbf{N}$ for which

$$
T^{-1}(0)+T^{k}(X) \text { is closed and } T^{-1}(0) \cap T^{k}(X) \text { is finite dimensional, }
$$


and if $S, S^{\prime}$ are invertible, commute with $T$ and have sufficiently small norm, then we have

$$
\operatorname{dim}(T-S)^{-1}(0)=\operatorname{dim}\left(T-S^{\prime}\right)^{-1}(0)<\infty,
$$

and

If in addition

4.4

$$
T^{-1}(0)+T^{k}(X) \text { is of finite codimension, }
$$

then also

$$
\operatorname{dim} X /(T-S) X=\operatorname{dim} X /\left(T-S^{\prime}\right) X<\infty .
$$

Proof. (2.2), with $\left(T, T^{k}\right)$ in place of $(S, T)$, says that $T^{k}(X)$ is closed; also (2.1) and (3.1) with $S=T^{k+m}(m=0,1,2, \ldots)$ gives the inductive step, which shows that $T^{n}(X)$ is closed for all $n \geq k$. Hence also the hyperrange $T^{\infty}(X)$ is closed and therefore complete. It follows that, since also $T^{\wedge}$ is onto, the operator $(T-S)^{\wedge}$ is onto for $S$ with sufficiently small norm commuting with $T$. At the same time, if $S$ is invertible and commutes with $T$, then the null space of $(T-S)^{\wedge}$ is the same as that of $T-S$. Thus if $S$ is invertible, commutes with $T$ and is of sufficiently small norm, then $(T-S)^{-1}(0)$ is finite dimensional and we can write

4.6

$$
\operatorname{dim}(T-S)^{-1}(0)=\operatorname{dim}\left((T-S)^{\wedge}\right)^{-1}(0)=\operatorname{index}\left((T-S)^{\wedge}\right)=\operatorname{index}\left(T^{\wedge}\right),
$$

using the continuity of the Fredholm index on the Banach space $T^{\infty}(X)$. This shows that the dimension of the null space of $T-S$ is to a certain extent independent of $S$, which is the first part of (4.2). For the closedness of the range of $T-S$ observe that the restriction of $T$ to $T^{n}(X)$ is also upper semi-Fredholm for each $n \geq k$, and hence also the restriction of $T-S$ for $S$ of sufficiently small norm commuting with $T$. Now

$4.7 \quad T^{-k}(T-S) T^{k}(X)=T^{-k} T^{k}(T-S)(X)=(T-S)(X)+T^{-k}(0)=(T-S)(X)$.

The third equality is obtained by interchanging the roles of $T$ and $T-S$ in (0.4), the second is elementary linear algebra and the first is because $T-S$ commutes with $T$. Since $(T-S) T^{k}(X)$ is closed, so is its counterimage by $T^{k}$. Towards (4.5), observe that $T^{-1}(0)+T^{k}(X) \subseteq T^{-k}(0)+T(X)$. Thus by (3.1) and (4.4), $T^{-k}(0)+T(X)$ is closed and of finite codimension. Now we claim that condition (4.4) is sufficient for the dual operator $T^{\dagger}$ on $X^{\dagger}$ to satisfy condition (4.1). By another argument of Kato [6, (4.12) Theorem [V.4.8]

$$
S^{-1}(0)+T(X) \text { closed } \Rightarrow S^{-1}(0)^{\perp}+T(X)^{\perp} \text { closed, }
$$

while by the theorem of Banach [6 (4.11) Theorem IV.4.8]

$$
S(Y) \text { closed } \Rightarrow S^{-1}(0)^{\perp}+T(X)^{\perp}=\left(T^{\dagger}\right)^{-1}(0)+S^{\dagger}\left(Z^{\dagger}\right)
$$

and

$$
S(Y) \text { closed } \Rightarrow\left(S^{-1}(0)+T(X)\right)^{\perp}=S^{\dagger}\left(Z^{\dagger}\right) \cap\left(T^{\dagger}\right)^{-1}(0) .
$$

For an alternative derivation of the closedness of the range compare Grabiner [2 Lemma 4.2, Theorem 4.7]. The closedness of the ranges of powers of $T$ under the 
conditions (4.1) can also be deduced from a result of Saphar [11, Proposition 7]. Taking $k=1$ in (4.1) shows that $T^{2}(X)$ is closed, so that (4.2) and (4.3) give back Schmoeger's result [12, Theorem 1].

Theorem 4 incorporates a corrected version of Theorem 2 of [9], in which the first part of condition (4.1) is omitted. Unfortunately this doesn't work.

5. EXAMPLE. If $Y=l_{p}$ or $Y=c_{0}$ and

5.1

$$
T=\left(\begin{array}{ll}
W & 0 \\
I & 0
\end{array}\right):\left(\begin{array}{l}
Y \\
Y
\end{array}\right) \rightarrow\left(\begin{array}{l}
Y \\
Y
\end{array}\right)
$$

with

5.2

$$
W\left(y_{1}, y_{2}, y_{3}, \ldots\right)=\left(y_{1}, \frac{1}{2} y_{2}, \frac{1}{3} y_{3}, \ldots\right)
$$

then

$$
\begin{gathered}
T=T T^{\prime} T \text { with } T^{\prime} \in B L(X, X) \text { is regular, } \\
T^{-1}(0) \cap T(X)=T^{-\infty}(0) \cap T(X)=\{0\}
\end{gathered}
$$

and

5.5

$$
T^{2}(X) \neq \operatorname{cl} T^{2}(X)
$$

Also

$$
\operatorname{dim}\left(T-\frac{1}{n} I\right)^{-1}(0)=1 \quad \text { for each } n \in \mathbf{N}
$$

and

$$
\operatorname{dim}(T-\lambda I)^{-1}(0)=0 \quad \text { if } \quad 0 \neq \lambda \notin\left\{\frac{1}{n}: n \in \mathbf{N}\right\}
$$

Proof. We have

$$
T=T T^{\prime} T \quad \text { with } \quad T^{\prime}=\left(\begin{array}{ll}
0 & I \\
I & 0
\end{array}\right),
$$

giving the "regularity" (5.3), and a straightforward calculation gives (5.4). It is clear also that

$$
T^{2}(X)=\left\{\left(W^{2} y, W y\right): y \in Y\right\}
$$

is not closed; also (5.6) and (5.7) are easily verified.

The operator $T$ of (5.1) satisfies the second part of condition (4.1), but by (5.6) and (5.7) fails the "punctured neighbourhood" property of Theorem 2 from [9]; it also fails the conclusion of Lemma 5 of [8].

Theorem 4 gives a genralization of the "Riesz-Schauder theorem" [13, Proposition $2.6,2.7]$ for semi-Fredholm operators: if in addition to the condition (4.1) $T$ is "of finite ascent", in the sense that for some $k \in \mathbf{N}$ the null spaces of $T^{k}$ and $T^{k+1}$ coincide, then 0 cannot be an accumulation point of the approximate point spectrum of $T$. This is clear from the proof of Theorem 4, remembering [3, (6.5.4.6), (7.8.4.1)], the isomorphism

$$
(S T)^{-1}(0) / T^{-1}(0) \cong S^{-1}(0) \cap T(X) \text {, }
$$


which says that the finite ascent condition specialises the second half of the condition (4.1). By (4.6) and (4.7) the operator $T-S$ is one-to-one with closed range for invertible $S$ of sufficiently small norm commuting with $T$. We have been unable to decide whether (4.2) and (4.5) still follow if (4.4) and the second part of (4.1) are replaced by the condition

$$
T^{-1}(0) \cap T^{k}(X)=T^{-1}(0) \cap T^{n}(X) \text { is closed for all } n \geq k .
$$

This certainly works if $X$ is a Hilbert space [10], or more generally if the condition "closed" in (4.1) and (5.10) is replaced by "closed and complemented".

\section{REFERENCES}

1. S. Goldberg, Unbounded linear operators (McGraw-Hill, New York, 1996).

2. S. Grabiner, Uniform ascent and descent of bounded operators, J. Math. Soc. Japan 34 (1982), 317-337.

3. R. E. Harte, Invertibility and singularity (Dekker, New York, 1988).

4. R. E. Harte, On Kato non-singularity, Studia Math. 117 (1996), 107-114.

5. T. Kato, Perturbation theory for nullity, deficiency and other quantities of linear operators, J. Analyse Math. 6 (1958), 261-322.

6. T. Kato, Perturbation theory for linear operators (Springer-Verlag, 1976).

7. K. B. Laursen and M. Mbekhta, Closed range multipliers and generalized inverses, Studia Math. 107 (1993), 127-135. $185-189$.

8. W. Y. Lee, Boundaries of the spectra in $L(X)$, Proc. Amer. Math. Soc. 116 (1992),

9. W. Y. Lee, A generalization of the punctured neighborhood theorem, Proc. Amer. Math. Soc. 117 (1993), 107-109. 69-105.

10. M. Mbekhta, Résolvant géneralisé et théorie spectrale, J. Operator Theory 21 (1989),

11. P. Saphar, Contribution a l'étude des applications linéaires dans un espace de Banach, Bull. Soc. Math. France 92 (1964), 363-384.

12. C. Schmoeger, On a generalized punctured neighbourhood theorem in $L(X)$, Proc. Amer. Math. Soc. 123 (1995), 1237-1240.

13. T. T. West, A Riesz-Schauder theorem for semi-Fredholm operators, Proc. Roy. Irish Acad. Sect. A 87 (1987), 137-146.

School of Mathematics

TRINITY COLlEge

DUBLIN 2

IRELAND
Department of Mathematics

SUNG KWAN UNIVERSITY

SUWON 440-746

KOREA 